\title{
Treatment selection for gastric cancer with portal hypertension: clinical management
}

\author{
Zhou-Xiang Jin $\cdot$ Yong-Yong Ma $\cdot$ Xiang-Yu Wang $\cdot$ \\ Li-Jun Li $\cdot$ Zhi-Qiang Zheng
}

Received: 4 February 2013/Accepted: 28 May 2013/Published online: 28 June 2013

(C) The International Gastric Cancer Association and The Japanese Gastric Cancer Association 2013

\begin{abstract}
Background Treatment for gastric cancer with portal hypertension must consider the eradication of the tumor and the change of hemodynamics in portal hypertension (PHT). Few reports have described the surgical procedures and postoperative complications of surgery for gastric cancer associated with PHT.

Methods The clinical data of 22 patients with PHT undergoing curative surgery for gastric cancer during 5 years were retrospectively analyzed. For 12 patients classified in Child's class A, D2 lymph node (LN) dissection was performed, and 10 patients classified into Child's class B were treated with D1 LN dissection. Surgical treatment included total gastrectomy combined with pericardial devascularization, distal subtotal gastrectomy, distal subtotal gastrectomy combined with splenectomy, and distal subtotal gastrectomy combined with pericardial devascularization with posterior gastric artery and left inferior phrenic artery preserved. A liver biopsy was analyzed in all patients.

Results Postoperative complications developed in $50 \%$ (11/22 patients) and the mortality rate was $9 \%(2 / 22)$. The rate of postoperative ascites in patients with Child's class A
\end{abstract}

Z.-X. Jin and Y.-Y. Ma contributed equally to this work.

Z.-X. Jin $(\bowtie) \cdot$ X.-Y. Wang · L.-J. Li · Z.-Q. Zheng

Department of General Surgery, Gastric Cancer Research

Center, The Second Affiliated Hospital of Wenzhou Medical

College, 109\#, XueYuan Western Road, Wenzhou 325027,

People's Republic of China

e-mail: jzx0847@sina.com.cn

Y.-Y. Ma

Department of Hematology, The First Affiliated Hospital of Wenzhou Medical College, Wenzhou, Zhejiang 325027, People's Republic of China was much lower than in those with Child's class B $(P<0.05)$. "Operation time," "volume of hemorrhage," "platelet count," and "treatment of PHT" are all risk factors of liver function deterioration. However, there was no significant difference in liver function deterioration rate between patients with Child's class A and Child's class B $(P>0.05)$. The occurrence rate of complications in patients with PHT was much higher compared to those without with PHT $(P<0.05)$.

Conclusions Individualized selection of surgical approaches is crucial for treatment of gastric carcinoma accompanied by PHT. Surgical treatment should be based on preoperative TNM stage, liver function, and degree of PHT.

Keywords Gastric cancer · Portal hypertension - Surgery approaches

\section{Introduction}

Gastric carcinoma (GC) is still one of the most frequently diagnosed cancers and the second most common cause of death from cancer worldwide [1]. Another major health problem in China is liver cirrhosis (LC), because of the high prevalence of hepatitis B virus (HBV) infection. Therefore, LC is not infrequently encountered among candidates for gastric cancer surgery [2]. A gastrectomy with D2 lymphadenectomy is the standard surgical procedure for gastric cancer because lymph node metastasis is an important prognostic factor, as is the depth of primary tumor invasion [3]. However, such an extended lymphadenectomy in gastric cancer patients with LC can lead to severe postoperative morbidity and mortality, and especially massive ascites $[4,5]$. Because LC itself is 
considered to be a terminal stage of liver disease, determining the appropriate surgical strategy in patients with gastric cancer combined with LC is controversial. Postoperative massive ascites is the most dangerous complication after an extended lymphadenectomy. Hyun et al. [6] reported that the rates of postoperative complications and mortality are 39 and $9 \%$, respectively. Thus, treatment of gastric cancer with portal hypertension (PHT) must consider the eradication of the tumor and change of hemodynamics in PHT. However, few reports have described the surgical procedures and postoperative complications of operations performed for gastric cancer associated with PHT. We summarized the clinical records of 24 patients diagnosed as gastric carcinoma combined with PHT between January 2003 and June 2008 and explored the selection of treatment by operation.

\section{Materials and methods}

\section{Patients}

A total of 1,347 consecutive patients underwent gastrectomy for GC at the Department of General Surgery, The Second Affiliated Hospital of Wenzhou Medical College, between January 2003 and June 2008. Of the 1,347 GC patients, $24(1.8 \%)$ were diagnosed as having PHT, and these 24 patients were enrolled in this study. Patients for the present study included 14 men and 10 women with a median age of 56.4 years (range 46-68 years).

Diagnosis and stage of gastric carcinoma: esophagogastroduodenoscopy with biopsy, abdominal computerized tomographic (CT) examination, plain chest X-ray, contrastenhanced ultrasound, and basic laboratory blood tests.

\section{Diagnosis of liver cirrhosis and PHT}

Liver cirrhosis was diagnosed according to clinical data in addition to either a positive radiologic or pathological result (i.e., CT scan, nuclear magnetic resonance imaging, ultrasonography, endoscopy, or liver biopsy). An intraoperative liver biopsy was performed in 22 of the 24 patients in our study. All the laboratory testing was performed with commercial kits at the routine hospital laboratory. Persistent infection with hepatitis B virus (HBV) and hepatitis C virus (HCV) was assessed by evaluating the hepatitis $\mathrm{B}$ surface antigen (HBsAg) and anti-HCV antibodies in serum. PHT was defined by the increase of hepatic vein pressure gradient (HVPG) or free portal pressure (FPP) above a threshold value of about $10 \mathrm{mmHg}$ or $25 \mathrm{cmH}_{2} \mathrm{O}$, respectively. The presence of esophageal-gastric varices, hypersplenism, variceal bleeding, or ascites indicates the presence of PHT.
Preoperative assessment of liver damage

Initially, laboratory measurements including aspartate aminotransferase (AST), alanine aminotransferase (ALT), alkaline phosphatase, platelets, serum creatinine, serum albumin, direct and total bilirubin, and coagulation tests were made for all patients. Ultrasound for the detection of ascites and endoscopy for varices were performed for all patients. To assess the severity of liver damage, the ChildPugh classification was used [7]. Indocyanine green (ICG) disappearance rate was also determined and used as an indicator of hepatic functional reserve. After intravenous injection of ICG $(0.5 \mathrm{mg} / \mathrm{kg})$, plasma ICG disappearance rate (K-ICG) was calculated by linear regression from plasma ICG concentrations at 5, 10, and $15 \mathrm{~min}$.

Preoperative assessment of tumor stage

The clinicopathological data were evaluated according to the General Rules for Gastric Cancer Staging in Surgery and Pathology established by the Japanese Research Society for Gastric Cancer [8]. Early cancer was defined as a T1 tumor and advanced cancer was defined as $\geq \mathrm{T} 2$ tumor, regardless of nodal involvement.

\section{Tumor and selection of surgery}

Total or distal gastrectomy was selected depending on the location and the macroscopic type of GC. When the tumor was located in the middle or upper third of the stomach, a total gastrectomy was performed. When the cancer-free margin could not be ensured by a distal gastrectomy because of an infiltrative growth pattern, a total gastrectomy was selected regardless of the main location of the tumor. For gastric carcinoma located in the lower third, and for those without need of treatment of PHT, distal gastrectomy was first favored. Concurrent with handling PHT, three surgical methods were chosen [1]: distal gastrectomy combined with splenectomy [2]; total gastrectomy with reservation of left inferior phrenic artery and posterior gastric artery [3]; and total gastrectomy combined with pericardial devascularization.

Curative surgery was defined as resection with no apparent residual tumors. In curative surgery for gastric cancer, the degree of LN dissection was scored according to the Japanese Classification of Gastric Carcinoma: D1 is limited $\mathrm{LN}$ dissection in which the perigastric $\mathrm{LNs}$ are removed; and D2 is extended $\mathrm{LN}$ dissection in which LNs along the common hepatic artery, along the splenic artery, and around the celiac axis are also removed [9]. In our study, 12 cases with Child's class A underwent D2 lymph node dissection and 10 cases who had Child's class B underwent D1 lymph node dissection. 


\section{Treatment of PHT}

Concurrent treating of PHT with resection of GC is still controversial. In our study, for patients with need of concurrent treating of PHT and resection of GC, the following prerequisites are required: (a) patient in good condition, with no severe heart, lung, kidney, or other important organ lesions; (b) gastric lesions can be radically removed; and (c) patient had good hepatic functional reserve (Child's class A or B). The surgical choice of PHT was decided by risk of upper gastrointestinal bleeding of patients and extent of hypersplenism. Indications for resection of gastric carcinoma with splenectomy (or splenic artery ligation) generally included (a) splenomegaly (major diameter $>12 \mathrm{~cm}$ ) with platelet count $<60 \times 10^{9} / 1$ and white blood cell count $<3.0 \times 10^{9} / 1$, according to the Barcelona Clinic Liver Cancer (BCLC) group criteria; (b) hemorrhagic tendency owing to hypersplenism observed by clinical manifestation or laboratory examination results. A total devascularization operation was indicated, such as pericardial devascularization, if the following high-risk factors existed: (a) splenomegaly, hypersplenism combined with esophageal or/and gastric varices of severe varicosity; (b) upper gastrointestinal bleeding history (recurrent bleeding was defined as any episode of upper gastrointestinal tract bleeding occurring after the first successful sclerotherapy session or subsequently between scheduled treatment sessions); and (c) preoperative measurement of free portal pressure (FPP) $\geq 42 \mathrm{cmH}_{2} \mathrm{O}$.

Preoperatively FPP was measured via venous cannulation of the right gastroepiploic vein. Average FPP was $36.0 \pm 4.8 \mathrm{cmH}_{2} \mathrm{O}$ (range $\left.29-47 \mathrm{cmH}_{2} \mathrm{O}\right) \quad\left(1 \mathrm{cmH}_{2} \mathrm{O}=\right.$ $0.098 \mathrm{kPa}$ ). Average operation time was $190.4 \pm 35.1 \mathrm{~min}$ (range 150-300 min. Average blood loss during operation was $779.5 \pm 284.7 \mathrm{ml}$ (range 300-1,500) ml. Liver biopsy was performed in all surgical cases.

\section{Preoperative preparation}

Before operation, we initiated liver protective therapy, supplied correct blood volume, and maintained the stability of water electrolyte values and acid-base balance. Correction of anemia, hypoproteinemia, and blood coagulation dysfunction was also indicated. Diuretic therapy was indicated if a patient complained of abdominal distension and if body weight increased more than $1 \mathrm{~kg}$ per day or abdominal circumference increased $5 \mathrm{~cm}$ per day. After treatment for 1-2 weeks, Child-Pugh classification was evaluated: 12 cases were categorized as Child's class A, 10 cases as class B, and 2 cases as class C. In all, 22 cases underwent surgical operation; all were categorized as Child's class A or B and classified as TNM stage I-III. Of these, 2 cases (Child's class C) were under conservative treatment, of which one was at the stage TNM IV and the other at the stage TNM III.

Postoperative complications and mortality

The occurrence of postoperative complications including ascites, anastomosis leakage, left inferior phrenic sepsis, pleural effusion, hepatic hepatorenal syndrome, and mortality was recorded in every patient. The postoperative Child-Pugh classification in every patient was reevaluated. The occurrence rate of postoperative complications including ascites, anastomosis leakage, left inferior phrenic sepsis, pleural effusion, hepatic hepatorenal syndrome, and mortality was compared between patients with Child's class A and class B. The risk factors of liver function deterioration and postoperative complications were also evaluated.

\section{Statistical treatment}

Results are presented as median (interquartile range), and nonparametric statistical testing was used. The data were analyzed using an SPSS statistical program. The $\chi^{2}$ test was used to compare categorical variables between groups. A survival curve was made with log-rank analysis.

Statistical significance was defined as $P<0.05$.

\section{Results}

Patient characteristics are seen in Table 1.

Changes of FPP of patients after devascularization operation

The average FPP in patients with additional devascularization before operation was $43.76 \pm 1.28 \mathrm{cmH}_{2} \mathrm{O}$. After operation the average FPP was $36.35 \pm 2.42 \mathrm{cmH}_{2} \mathrm{O}$, which was significantly decreased $(P \leq 0.05)$.

Postoperative complications and mortality

Among the 22 patients, $11(50 \%)$ developed postoperative complications, including ascites, anastomosis leakage, left inferior phrenic sepsis, pleural effusion, hepatic hepatorenal syndrome (HRS), wound infection, and mortality (Table 2). The incidence of pleural effusion, hepatorenal syndrome, wound infection, and left inferior phrenic sepsis between patients with Child's class A and Child's class B was not significantly different $(P>0.05)$. However, the incidence of ascites in patients with Child's class A was much lower than in those with Child's class $\mathrm{B}(P<0.05)$. 
Table 1 Patient characteristics

\begin{tabular}{|c|c|c|c|}
\hline Factor & $\begin{array}{l}\text { Child's class A } \\
(n=12)\end{array}$ & $\begin{array}{l}\text { Child's class B } \\
(n=10)\end{array}$ & $\begin{array}{l}\text { Child's class C } \\
(n=2)\end{array}$ \\
\hline \multicolumn{4}{|l|}{ Age (years) } \\
\hline$\leq 55$ & 7 & 6 & 1 \\
\hline$>55$ & 5 & 4 & 1 \\
\hline \multicolumn{4}{|l|}{ Sex } \\
\hline Male & 8 & 5 & 1 \\
\hline Female & 4 & 5 & 1 \\
\hline \multicolumn{4}{|l|}{ Cause of cirrhosis } \\
\hline HBV related & 10 & 9 & 1 \\
\hline HCV related & 1 & 0 & 1 \\
\hline Alcohol & 1 & 1 & 0 \\
\hline \multicolumn{4}{|l|}{ Location of tumor } \\
\hline Upper third & 3 & 1 & 1 \\
\hline Middle third & 1 & 5 & 1 \\
\hline Lower third & 8 & 4 & \\
\hline \multicolumn{4}{|l|}{ Tumor stage } \\
\hline Stage I & 2 & 1 & 0 \\
\hline Stage II & 5 & 6 & 0 \\
\hline Stage III & 5 & 3 & 1 \\
\hline Stage IV & 0 & 0 & 1 \\
\hline \multicolumn{4}{|l|}{ Operative approach } \\
\hline Total gastrectomy + pericardial devascularization & 5 & 6 & \\
\hline Distal subtotal gastrectomy & 6 & 3 & \\
\hline $\begin{array}{l}\text { Distal subtotal gastrectomy }+ \text { splenectomy or } \\
\text { splenic artery ligation }\end{array}$ & 1 & & \\
\hline $\begin{array}{l}\text { Distal subtotal gastrectomy }+ \text { pericardial } \\
\text { devascularization }+ \text { with reservation of left } \\
\text { inferior phrenic artery and posterior gastric artery }\end{array}$ & & 1 & \\
\hline Extent of lymphadenectomy & $\mathrm{D}_{2}$ & $\mathrm{D}_{1}$ & \\
\hline History of episodes of UGI bleeding & 4 & 2 & 2 \\
\hline
\end{tabular}

Table 2 Postoperative morbidities and mortalities according to Child-Pugh classification

\begin{tabular}{llll}
\hline & $\begin{array}{l}\text { Child's class A } \\
(n=12)\end{array}$ & $\begin{array}{l}\text { Child's class B } \\
(n=10)\end{array}$ & $P$ value \\
\hline Ascites & 2 & 6 & 0.035 \\
Pleural effusion & 1 & 3 & 0.075 \\
Hepatorenal syndrome & 0 & 1 & 0.209 \\
Anastomotic leakage & 0 & 1 & 0.209 \\
Wound infection & 2 & 2 & 0.924 \\
$\quad$ Left inferior phrenic & 1 & 1 & 0.830 \\
$\quad$ sepsis & & 2 & 0.104 \\
$\quad$ Mortality & 0 & &
\end{tabular}

Two patients (9\%) died postoperatively, one from hepatic hepatorenal syndrome and the other from postoperative anastomosis leakage after distal gastrectomy (dissection of lymph node $\mathrm{D}$ with pericardial devascularization reservation of the left inferior phrenic artery). None of the patients with Child's class A died of complications. Average postoperative hospital stay (days) was 38.8 days (range 25-51 days).

Factors influencing liver function and postoperative complications

Operation time, volume of hemorrhage, platelet count, and treatment of PHT were all risk factors of liver function deterioration. However, there was no significant difference in liver function deterioration rate between patients with Child's class A and Child's class B $(P>0.05)$. Volume of hemorrhage, Child-Pugh classification, indocyanine green retention rate at $15 \mathrm{~min}$ (ICGR15), and treatment of PHT were all risk factors of occurrence of postoperative complications. The incidence of complications in patients with PHT was much higher compared to those without PHT $(P<0.05)$ (Table 3). 
Table 3 Change of liver function class and incidence of postoperative complications

\begin{tabular}{|c|c|c|c|c|c|c|}
\hline \multirow[t]{2}{*}{ Factors } & \multicolumn{3}{|c|}{ Change of liver function grade } & \multicolumn{3}{|c|}{ Postoperative complication } \\
\hline & Stability $(n=10)$ & Deterioration $(n=12)$ & $P$ value & Yes $(n=11)$ & No $(n=11)$ & $P$ value \\
\hline \multicolumn{7}{|c|}{ Age (years) } \\
\hline$\leq 55$ & 6 & 7 & 0.937 & 6 & 7 & 0.665 \\
\hline$>55$ & 4 & 5 & & 5 & 4 & \\
\hline \multicolumn{7}{|c|}{$\mathrm{ICG}_{\mathrm{R} 15}(\%)$} \\
\hline$\leq 10$ & 2 & 1 & 0.611 & 0 & 3 & 0.03 \\
\hline $10-20$ & 4 & 4 & & 3 & 5 & \\
\hline$\geq 20$ & 4 & 7 & & 8 & 3 & \\
\hline \multicolumn{7}{|c|}{ Diameter of tumor $(\mathrm{cm})$} \\
\hline$\leq 3$ & 7 & 4 & 0.198 & 3 & 8 & 0.086 \\
\hline$>3$ & 3 & 8 & & 8 & 3 & \\
\hline \multicolumn{7}{|c|}{ Platelet count $\left(10^{9} / 1\right)$} \\
\hline$\leq 100$ & 4 & 11 & 0.02 & 6 & 9 & 0.361 \\
\hline$\geq 100$ & 6 & 1 & & 5 & 2 & \\
\hline \multicolumn{7}{|c|}{ Operation time (min) } \\
\hline$\leq 180$ & 8 & 2 & 0.008 & 3 & 7 & 0.198 \\
\hline$>180$ & 2 & 10 & & 8 & 4 & \\
\hline \multicolumn{7}{|c|}{ Volume of hemorrhage (ml) } \\
\hline$\leq 800$ & 9 & 2 & 0.002 & 2 & 9 & 0.009 \\
\hline$>800$ & 1 & 10 & & 9 & 2 & \\
\hline \multicolumn{7}{|c|}{ Child's class } \\
\hline A & 7 & 5 & 0.231 & 3 & 9 & 0.03 \\
\hline B & 3 & 7 & & 8 & 2 & \\
\hline \multicolumn{7}{|c|}{ Treatment of PHT } \\
\hline Yes & 3 & 10 & 0.027 & 10 & 3 & 0.008 \\
\hline No & 7 & 2 & & 1 & 8 & \\
\hline
\end{tabular}

\section{Long-term outcomes}

Except for 2 patients who died, the other 22 patients were followed as outpatients or by telephone follow-up: 18 patients died after 1-3 years, 8 of hepatic failure, 4 of upper gastrointestinal bleeding, and 6 of recurrence of gastric cancer. Follow-up included endoscopy (if deemed necessary by the endoscopist), and clinical and laboratory evaluation at 1 month postoperatively, every 6 months for the first 5 years, and every year thereafter. Those patients who were not able to attend the clinic regularly were contacted by telephone or through questionnaire sent by mail. The survival curve of patients with Child's class A or $\mathrm{B}$, respectively, is seen in Fig. 1. The survival time of patients with Child's class A was significantly longer than that of patients with Child's class B $(P<0.05)$.

\section{Discussion}

It is well known that patients with LC have a high incidence of postoperative complications and postoperative mortality [10-13]. Hyun et al. [6] reported that the rate of postoperative complications and mortality is $39 \%$ and $9 \%$, respectively. In our study, the 24 patients were a subset of more than 1,300 patients. The incidence of gastric carcinoma complicated by PHT was infrequent in our study, so management of these patients remained a challenge. Recent advances in surgical techniques and postoperative management have reduced the morbidity and mortality associated with gastric cancer surgery. However, there have been a limited number of reports describing postoperative complications in patients with LC who undergo radical surgery for gastric cancer. Therefore, during the perioperative period patients should be comprehensively evaluated and individually treated.

Preoperative evaluations were as follows. (a) Evaluation or resectability of gastric carcinoma: preoperative gastroscope and computerized tomographic examination were done to determine the location and size of the tumor and possible distant metastasis. In our hospital, gastroduodenal double-contrast ultrasound was used to confirm the location of the lesion, depth of invasion, and scope [14]. Contrastenhanced ultrasound of the liver was further used to help find intrahepatic occult metastatic cells [15]. (b) Preoperative evaluation of liver function was done in all patients. 
Fig. 1 Survival curves (cumulative, cum) of patients with Child A or Child B classification

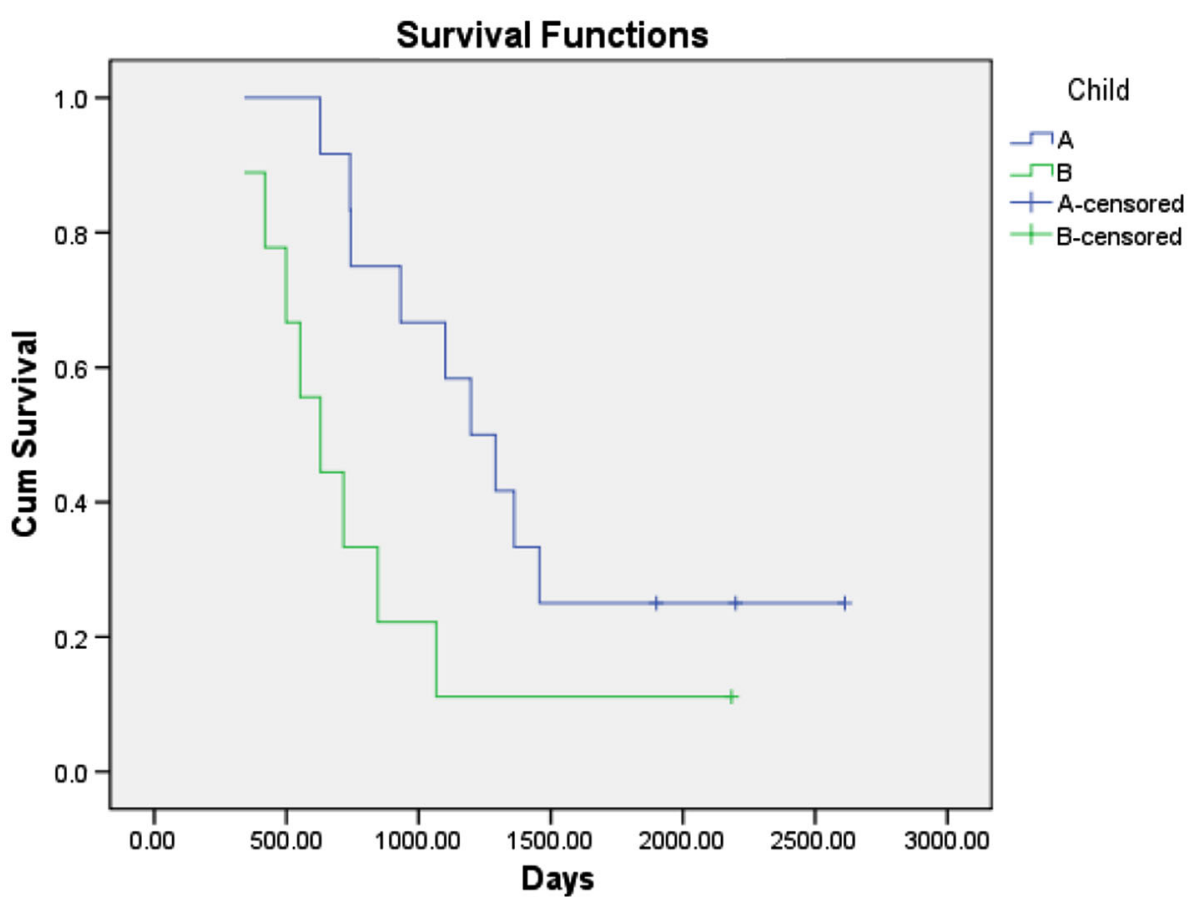

Preoperative liver function was assessed according to the Child-Pugh classification, but the disadvantage of this was the situation of hepatic dysfunction and quiescent condition degree of compensation. (c) Evaluation of portal hypertension: preoperative measurement of superior mesenteric, maximum portal flow velocity, and blood flow direction with color Doppler ultrasonography. Extent of splenomegaly with or without hypersplenism and volume of ascites were further evaluated.

Gastric cancer surgery in our institution followed standardized operative protocols: a total or distal gastrectomy was selected depending on the location and the macroscopic type of GC. When the tumor was located in the middle or upper third of the stomach, a total gastrectomy was performed. When the cancer-free margin could not be ensured by a distal gastrectomy because of an infiltrative growth pattern, a total gastrectomy was selected regardless of the main location of the tumor. For gastric carcinoma located in the lower third, the choice of treatment for distal third of the stomach gastric cancer remains controversial. Reports suggest that if a distal subtotal gastrectomy with regional lymphadenectomy, a standard operation for gastric cancer, is performed, then the esophageal and/or gastric varices may deteriorate quickly, thus leading to a rupture that can often be fatal. This complication occurs because the residual hepatofugal collateral pathways, which flow via the residual short gastric veins and posterior gastric veins from the esophageal and/or gastric varices directly through the blood vessels of the gastric wall after the major hepatofugal collateral pathways, such as the left gastric vein, have been resected with the stomach [16]. On the other hand, deterioration of esophageal varices has also been reported to occur after the operation when total gastrectomy has been performed, and all the collateral pathways have been devascularized, because a large portal vein flow had been directed into the esophageal wall directly through the blood vessels of the Roux-en-Y loop [17]. Thus, the surgical choice for distal gastric carcinoma must be fully considered. In our study, for those without need of treatment of PHT, distal gastrectomy was favored. Perspective follow-up found no rupture of esophageal or gastric varices. Concurrent with treating PHT, especially during gastroesophageal devascularization, the blood supply of the proximal stomach will be greatly affected. Some reports indicated that a distal gastrectomy combined with pericardial devascularization with reservation of the left inferior phrenic artery and superior gastric artery can avoid ischemia of the remnant stomach [18]. However, we contend that hypoplasia or the absence of the superior gastric artery already exists in some patients. Furthermore, the left inferior phrenic artery was far from the gastric remnant, which increased the ratio of fistula of anastomosis because of ischemia of the gastric remnant. Further, in the situation of severe perigastric varicosity and dense adhesions around the spleen, reservation of these vessels may increase the operative time and loss of blood, and even lead to incomplete devascularization. In our group, one patient died of anastomotic fistula after distal gastrectomy combined with pericardial devascularization with reservation of the left inferior phrenic artery and superior gastric artery. Therefore, we believe that total gastrectomy will be safer for patients with distal gastric carcinoma with concurrent 
treatment of PHT. In our group, one patient with distal gastric carcinoma underwent total gastrectomy without severe complications.

Although the causes of death for patients with Child's class A were most commonly related to the recurrence of gastric cancer, the most common causes for patients with Child's class B or C were cirrhosis related, such as hepatic failure or hepatoma, followed by postoperative complications [19]. These observations suggest that aggressive surgery for patients with gastric cancer combined with PHT should not be carried out with the sole aim of achieving a curative resection. For patients with moderate to severe hepatic dysfunction, D1 or less extensive LN dissection may be the more reasonable surgical procedure. Shi et al. [20] also believed that for patients with PHT, D2 LN dissection should not be routinely performed: D1 LN dissection was sufficient. In our group, between Child's class A patients experiencing D2 LN dissection and Child's class B patients experiencing D1 dissection, the deterioration rate of liver function showed no significant difference $(P<0.05)$. However, the incidence of complications of Child's class A was lower than Child's class B. The prognosis of PHT patients depends on liver function status but not the operative choice. So, we believe that for Child's class A patients, D2 LN dissection should be safe and feasible, but perioperative liver protection treatment is necessary. These results demonstrated that radical gastrectomy in LC patients at a compensated stage was feasible. The incidence of complications for Child's class B patients was 12.66 fold that of Child's class A patients, especially in the presence of ascites [21]. So, to increase the safety of the operation, the operative choice for Child's class B patients should be D1 LN dissection without residual tumor visible by naked eye.

As for Child's class C, surgery should not be undertaken unless the situation represents an emergency. Based on our experience concerning postoperative morbidities and mortalities in patients with Child's class B or C, we suggest that avoiding LN dissection along the hepatoduodenal ligament should be considered for patients with moderate or severe hepatic dysfunction, except for those in whom metastases are macroscopically evident in the LNs.

With the improvement of radioactive intervention, the mortality rate of patients with PHT after the first bleeding undergoing medical treatment has been decreasing. Agreement has been reached that prophylactic devascularization was not essential. For patients with gastric carcinoma and PHT, the surgical alternative is still controversial. We believe that radical resection combined with gastroesophageal devascularization can reduce portal venous flow, increase the blood flow of the hepatic artery, remove hypersplenism secondary to liver cirrhosis and portal hypertension, and alleviate hepatic bilirubin load, contributing to liver regeneration and liver function recovery.
In liver cirrhosis, because portal venous blood flow is reduced, maintenance of hepatic arterial blood flow and preserved hepatic arterial buffer response probably represent a beneficial mechanism for hepatic circulation that upgrades three lineages of blood cells and enhances postoperative antitumor immunity. For patients with need of concurrent treatment for PHT and resection of GC, the following prerequisites are needed: (a) the patient's general condition is good, with no severe heart, lung, kidney, and other important organ lesions; (b) gastric lesions can be radically removed; (c) good hepatic functional reserve (Child's class A-B). The surgical alternative for PHT was decided by risk of upper gastrointestinal bleeding and the extent of hypersplenism. Indications for resection of gastric carcinoma with splenectomy (or splenic artery ligation) generally included (a) splenomegaly (major diameter $>12 \mathrm{~cm}$ ) with a platelet count $<60 \times 10^{9} / 1$ and white blood cell count below $3.0 \times 10^{9} /$; and (b) hemorrhagic tendency owing to hypersplenism observed by clinical manifestation or laboratory examination results. Entire devascularization was indicated, such as pericardial devascularization, with the following highrisk factors: (a) splenomegaly, hypersplenism combined with esophageal or/and gastric varices severe varicosity, and (b) upper gastrointestinal bleeding history. Recurrent bleeding was defined as any episode of upper gastrointestinal tract bleeding occurring after the first successful sclerotherapy session or subsequently between scheduled treatment sessions; and (c) preoperative measurement of FPP $\geq 42 \mathrm{cmH}_{2} \mathrm{O}$.

Out study showed that the incidence of complications between patients with or without PHT was significantly different $(P<0.05)$. Although the concurrent treatment of PHT was beneficial to patients, the key to successful surgical treatment of a patient with gastric carcinoma associated with liver cirrhosis and portal hypertension was the access to strictly ruled indications and a reasonable surgical procedure.

Overall, the treatment of patient with gastric carcinoma associated with PHT was complex, with much controversy. Attaching importance to perioperative management was crucial to the success of the operative procedure. Preoperative hepatic function, preoperative ascites and tumor stage were factors affecting postoperative survival. In conclusion, radical gastrectomy could be conducted safely in GC patients with Child's class B cirrhosis based on the Child-Pugh classification. However, the extent of lymph node dissection should be minimized because grade B patients are at risk of postoperative intractable ascites.

\section{References}

1. Parkin DM, Bray F, Ferlay J, et al. Global cancer statistics, 2002. CA Cancer J Clin. 2005;55:74-108. 
2. Carrison RN, Cryer HM, Howard DA, et al. Clarification of risk factors for abdominal operations in patients with hepatic cirrhosis. Ann Surg. 1984;199:648-55.

3. Siewert JR, Kestlmeier R, Busch R, et al. Benefits of $D_{2}$ lymph node dissection for patients with gastric cancer and $\mathrm{pN} 0$ and $\mathrm{pN} 1$ lymph node metastases. Br J Surg. 1996;83:1144-7.

4. Isozaki $\mathrm{H}$, Okajima K, Ichinona $\mathrm{T}$, et al. Surgery for gastric cancer in patients with cirrhosis. Surg Today. 1997;27:17-21.

5. Kumagai K. Intractable ascites following surgery for gastric carcinoma. Dig Surg. 1998;15:236-40.

6. Hyun JJ, Kim JH, Song Hun Ho, et al. Clinical outcomes of patients with liver cirrhosis who underwent curative surgery for gastric cancer: a retrospective multi-center study. Dig Dis Sci. 2008;53:399-404.

7. Pugh RN, Murray-Lyon IM, Dawson JL, et al. Transection of the oesophagus for bleeding oesophageal varices. $\mathrm{Br} \mathrm{J}$ Surg. 1973;60:646-9.

8. Japanese Gastric Cancer Association. Japanese classification of gastric carcinoma. 13th ed. Tokyo: Kanehara; 1999.

9. Dent DM, Madden MV, Price SK. Randomized comparison of R1 and R2 gastrectomy for gastric carcinoma. Br J Surg. 1988;75: $110-2$.

10. Garrison RN, Cryer HM, Howard DA, et al. Clarification of risk factors for abdominal operations in patients with hepatic cirrhosis. Ann Surg. 1984;199:648-55.

11. Fekete F, Belghiti J, Cherqui D, et al. Results of esophagogastrectomy for carcinoma in cirrhotic patients. A series of 23 consecutive patients. Ann Surg. 1987;206:74-8.

12. Sirinek KR, Burk RR, Brown M, et al. Improving survival in patients with cirrhosis undergoing major abdominal operations. Arch Surg. 1987;122:271-3.
13. Jakab F, Ráth Z, Sugár I, et al. Complications following major abdominal surgery in cirrhotic patients. Hepatogastroenterology. 1993;40:176-9.

14. Huang PT, Li YP, Zhao YP, et al. Diagnostic value of double contrast-enhanced ultrasonography in preoperative staging of gastric cancer. Chin Ultrasound Imaging J. 2008;1:740-2.

15. Huang PT, Zhao YP, Zheng ZQ, et al. Assessment of gray-scale contrast enhanced ultrasound on liver metastasis of advanced gastric carcinoma. Chin Ultrasound Imaging J. 2009;18:840-2.

16. Isozaki $\mathrm{H}$, Okajima $\mathrm{K}$, Ichinona $\mathrm{T}$, et al. Surgery for gastric cancer in patients with cirrhosis. Surg Today. 1997;27:17-21.

17. Chikamori F, Shibuya S, Takase Y. Percutaneous transhepatic obliteration for esophagojejunal varices after total gastrectomy. Abdom Imaging. 1998;23:560-2.

18. Zhou YB, Li SK, Zhang JL, et al. The surgical management of advanced gastric carcinoma with portal hypertension. Chin J Gen Surg. 2005;20:406-8.

19. Liu CL, Fan ST, Lo CM, et al. Abdominal drainage after hepatic resection is contraindicated in patients with chronic liver diseases. Ann Surg. 2011;239:194-201.

20. Shi CY, Zhou H, Wu ZY, et al. Surgery for gastric cancer with cirrhosis portal hypertension. Chin J Oper Proced Gen Surg. 2009;3:616-21.

21. Zhou BY, Chen SY, Wang H. Analysis of postoperative complications of gastric carcinoma with liver cirrhosis. Chin Surg J. 2008;23:950-2. 